\title{
The effect of visual orientation uncertainty in a simultaneous detection-recognition task*
}

\author{
STEVEN DOEHRMAN $\dagger$ \\ University of Michigan, Ann Arbor, Michigan 48104
}

\begin{abstract}
This study concerned the processing of the outputs of visual orientation-sensitive mechanisms by higher order mechanisms. Ss performed a simultaneous detection-recognition task. Variation in the number of possible orientations of a single straight line produced only slight, if any, changes in detection or recognition performance as compared with findings from similar auditory frequency experiments. It was concluded that the outputs of all orientation-sensitive mechanisms are examined by higher order mechanisms. As the angular separation of two possible orientations increased, the data showed that recognition improved in keeping with other reported findings. Comparison of detection with recognition indicated that the latter, under certain conditions, was superior to the former.
\end{abstract}

A number of studies (Campbell \& Kulikowski, 1966; Houlihan \& Sekuler, 1969; Sekuler, 1965; Antelman et al, 1969; Mayo et al, 1968; Gilinsky, 1968) have presented data suggesting functional mechanisms in the human visual system selectively sensitive to a restricted range of orientations. Although most of the studies show that the sensitivity of the hypothetical orientation-sensitive mechanisms extends $15.20 \mathrm{deg}$ either side of the preferred orientation, i.e., their range being 30-40 deg, other findings point to ranges of up to 90 deg (Parlee, 1969; also see Coltheart, 1971).

The present study deals with the processing of the outputs of orientation-sensitive mechanisms by higher order mechanisms. According to one model, the outputs of mechanisms sensitive to irrelevant orientations (according to specified stimulus conditions) are disregarded by higher order mechanisms. Further, an increase in the number of orientation-sensitive mechanisms that are not disregarded is assumed to allow more noise to be passed on to the higher order mechanisms. An alternative model might assert that the outputs of all orientation-sensitive mechanisms are examined by higher order mechanisms, regardless of the potential stimuli. The first model would predict that performance should diminish as the number of possible stimulus orientations increases. The second would predict no change in performance with variation of orientation uncertainty (OU).

Auditory frequency experiments have demonstrated performance changes with stimulus uncertainty. For

*This research was supported by funds from the National Science Foundation, Grant GB 8181, awarded to D. H. Krantz and $D$. J. Weintraub. This report is based on a dissertation which was submitted in partial fulfillment of the requirements for the Doctor of Philosophy (Psychology) degree in the Horace $H$. Rackham School of Graduate Studies at The University of Michigan. I wish to thank my doctoral chairman, D. H. Krantz, for his assistance and guidance in all phases of the research. I would also like to thank the other members of the doctoral committee: D. G. Green, W. M. Kincaid, and especially D. J. Weintraub.

+Now at the Psychological Clinic, 1027 East Huron Street, Ann Arbor, Michigan 48104. Send requests for reprints and for a more detailed description of the experiment to this address. example, Veniar (1958) found a decrease in detection performance with an increase in the number of possible frequencies. This result and others (see Green \& Swets, 1966, for a thorough discussion) have led to models of auditory "listening" bands $(50-500 \mathrm{~Hz}$ wide) which are sampled according to the number of possible stimulus frequencies.

In the present study, evidence was collected which deals with the involvement of "watching" bands in the detection and recognition of visual orientation. Change in recognition performance with variation in the range of possible orientations of a single line, expected in light of the studies cited earlier, serves as a comparison to any change in performance on either psychophysical task as a function of different amounts of orientation uncertainty. The data analyses also include a comparison of recognition performance with detection performance in the condition when both judgments are simultaneously required.

\section{METHOD}

\section{Subjects}

Three college women, aged 18-24, served as paid Ss. They were naive as to the purposes of the experiment. All had normal or corrected-to-normal visual acuity. The Ss' astigmatism was not determined.

\section{Apparatus and Stimulus Materials}

Two channels of a three-channel Scientific Prototype Model GB tachistoscope were used to present the stimulus materials. Each stimulus field was reduced in size to a circular area with a diameter of $4 \mathrm{deg} 20 \mathrm{~min}$ (visual angle). Stabilization of the S's head was achieved with a biteboard that was attached to a wooden frame mounted on the viewing hood. The frame also held in place Wratten neutral-density filters (density $=2.0$ ), which reduced the luminance of the fields without obstructing the binocular view of the S. Gilinsky and Doherty (1969) showed that dichoptic masking with line gratings produced an effect as a function of orientation which was not qualitatively different from that seen with monoptic masking. Thus, at least some orientation-sensitive mechanisms are at or beyond the cortical locus which receives input from both eyes. Hence, it was thought that, for visual orientation information, binocular 
viewing would not produce effects different from monocular viewing, and the former viewing condition was used in this experiment.

A Spectra brightness spot meter (Photo Research Corporation, Burbank, California) was used at the beginning of each session to set the luminance of the stimulus field at $0.57 \mathrm{~cd} / \mathrm{m}^{2}$ and the fixation field at $0.23 \mathrm{~cd} / \mathrm{m}^{2}$. The reading of the meter was calibrated with a MacBeth illuminometer.

Black straight line segments measuring $1 \mathrm{deg} 50 \mathrm{~min} \times 2.6 \mathrm{~min}$ (visual angle) were mounted on $12.7 \times 17.8 \mathrm{~cm}$ white index cards. Regardless of its orientation, the midpoint of a line segment coincided with the center of the circular stimulus field. The black fixation dot, subtending $4 \mathrm{~min}$, also appeared at this location. A blank white index card was used on no-signal or noise trials, i.e., trials on which no line was presented.

\section{Experimental Design}

The stimulus variables were:

(1) Pivot orientation (PO)-This is the reference or standard orientation from which the orientations of the stimulus lines deviated. Three PO conditions were used: horizontal, 1 vertical, and $45 \mathrm{deg}$ clockwise from vertical (oblique). The recognition response was based on the judged orientation of the "perceived" line with respect to a given PO, i.e., whether the line was oriented clockwise or counterclockwise with reference to the PO.

(2) $\alpha$-This is the distance in degrees that a line deviated from the PO. The absolute value of $\alpha$ was $3,7,16,29$, or $42 \mathrm{deg}$. The sign of $\alpha$ could be either positive or negative. Positive values refer to orientations clockwise from a specified PO. Such stimuli are designated $\mathrm{S}+$. Negative values refer to orientations counterclockwise from a specified $P O$, and such stimuli are designated S-. Large values of $\alpha$ in conjunction with the oblique PO are nearly horizontal or vertical, e.g., when $|\alpha|=42 \mathrm{deg}$ and PO is oblique, $S+$ is $3 \mathrm{deg}$, or almost horizontal, and $S-$ is $87 \mathrm{deg}$, or nearly vertical. Also, with $|\alpha|=42 \mathrm{deg}$ and PO horizontal, $\mathrm{S}+$ is $318 \mathrm{deg}$, almost $90 \mathrm{deg}$ from the oblique PO, and $S-$ is $42 \mathrm{deg}$, very close to the oblique PO. Both PO and the value of $\alpha$ are needed to specify the location of a stimulus line.

(3) Orientation uncertainty (OU)-On a signal trial, the line segment could be at one of one, two, or eight possible orientations, $\mathrm{OU}$ being $0,1,3$, respectively $\left(\mathrm{OU}=\log _{2}\right.$ number of possible line orientations). With OU of 3 , the eight possible orientations were $\pm 3, \pm 16, \pm 29, \pm 42 \mathrm{deg} ;|\alpha|=7 \mathrm{deg}$ was omitted. With OU of 1 , the two locations were equidistant from the pivot, with each of the five values of $|\alpha|$ used. With $O U$ of $0, \alpha=+7 \mathrm{deg}$ and $\alpha=-7 \mathrm{deg}$ were omitted.

\section{Responses}

The two types of responses were detection (D) and recognition ( $R)$. All the trials of a given session required $D$ only, $R$ only, $D$ followed by $R$, or $R$ followed by D. ${ }^{2}$ The probability of a line being presented on any trial of the experiment was 0.50 . With OU of 1 or 3 , the probability of $S+$ was equal to the probability of $\mathrm{S}-$, equal to 0.25 . Trial order was random, with the restriction that no more than four trials of any type could occur consecutively.

When required to make a detection response, the $S$ had to choose one of four confidence categories, ranging from high confidence of the signal's absence ("1") to high confidence of the signal's presence (" 4 "). When making a recognition response regarding the orientation of the line, the $S$ judged whether the tilt of the stimulus line was clockwise (high confidence $=$ "d," low confidence $=$ "c") or counterclockwise (high confidence $=$ " $a$," low confidence = " $b$ ") from the pivot orientation.

The Ss were instructed to spread both judgments among all four categories and to be more accurate with the high-confidence than with the low-confidence response, e.g., $P($ signal $\mid$ " 4 ") $>$ $\mathrm{P}($ signal | "3"), or $\mathbf{P}(\mathrm{S}+$ | "d") $>\mathbf{P}(\mathrm{S}+$ | "c").

No one $S$ was tested in all possible permutations of the stimulus variables and responses. Each $\mathrm{S}$ was given two $\mathrm{PO}$ conditions with four or five $\alpha$ levels each. C.A. received all three OU conditions, with N.A. and R.H. receiving two of three OU conditions.

\section{Procedure}

On each trial, the $\mathrm{S}$ centered her gaze on the fixation dot, and, upon the E's ready signal, initiated the test flash by pressing a hand-held microswitch. The fixation field returned with the offset of the test flash. There was a minimum of $8 \mathrm{sec}$ between every two trials. After each flash, the $S$ gave the required response (s). The $\mathrm{E}$ immediately gave feedback consisting of (1) "plus," i.e., a line segment clockwise from PO had been presented, (2) "minus," i.e,, a line segment counterclockwise from PO had been presented, or (3) "blank," i.e., no line segment had been presented. A brief rest period about 2 min long was taken after every 50 trials. Most sessions contained 400 trials and lasted about $90 \mathrm{~min}$.

During practice sessions, the exposure duration of the test flash was varied in order to achieve desired performance of roughly $75 \%$ correct. The nominal flash duration used for each $\mathrm{S}$ was: C.A., $11.5 \mathrm{~m} \mathrm{sec}$; N.A., $12.0 \mathrm{~m} \mathrm{sec}$; and R.H., $8.0 \mathrm{msec}$.

The Ss were informed at the beginning of the experiment of the stimulus presentation probabilities. At the beginning of each testing session, the $\mathrm{E}$ specified the $\mathrm{PO}$, the $\mathrm{OU}$, and responses (s) for all trials in that session. The only variable that took on more than one value in any session was $\alpha$.

\section{RESULTS}

The data were analyzed following the prescriptions of signal detectability theory (SDT). For detection, three values of the false alarm rate and of the hit rate were calculated by stepwise accumulation of frequencies in the response categories conditional upon the presented stimulus. Similarly, for recognition, the cumulative response proportions were found by progressive summing of the response categories for the two types of signal trials. (Recognition responses on noise trials were disregarded in the determination of recognition performance.) The resulting ROC points were used in determining ${ }^{3} \mathrm{~d}_{e}^{\prime}$ and $\mathrm{s}$, two performance measures of SDT defined by Green and Swets (1966, pp. 96-98). s for detection was generally well below 1.00 , around .60 to 80 , a fact in accordance with typical visual psychophysical findings (Nachmais \& Steinman, 1963; Rollman \& Nachmias, 1972). The results showed individual differences in the $d_{e}^{\prime}$ performance of the three Ss. R.H., with the smallest exposure duration, performed as well as C.A., both with detection $\mathrm{d}_{\mathrm{e}}^{\prime}$ in the neighborhood of 1.80 to 2.00 . N.A., with the longest exposure duration, performed the poorest, with detection $\mathrm{d}_{\mathrm{e}}^{\prime}$ around 1.00 . The relatively poor performance of N.A. was noted in the practice sessions, but since she used the response categories appropriately it was decided that there was no legitimate reason for excluding her from serving in the main experiment. The design provided a replication of some experimental conditions for C.A. and N.A. The data of C.A. did not change from the first to the second testing, whereas that of N.A. showed an inexplicable $30 \%$ decrease.

To examine the effect of OU, the $d_{e}^{\prime}$ data were analyzed using matched pairs $t$ tests. In each comparison, for each $S$, the detection or recognition $d_{e}^{\prime}$ 
Table 1

Comparisons of Performance (dé) Between Configurations [PO, OU, Required Response (s)] Which Had Different Values of OU

\begin{tabular}{|c|c|c|c|c|c|c|}
\hline $\mathrm{S}$ & Comparison & $\mathrm{t}$ & df & $\mathrm{p}$ & $\begin{array}{l}\text { Percent } \\
\text { Change }\end{array}$ & $\begin{array}{c}95 \% \\
\text { Confidence } \\
\text { Interval }\end{array}$ \\
\hline C.A. & $\begin{array}{l}\mathrm{V}, 0, \mathrm{D} \text { vs } \mathrm{V}, 1, \mathrm{D} \\
\mathrm{V}, 1, D \& \mathrm{R} \text { vs } \mathrm{V}, 3, D \& \mathrm{R} \\
\mathrm{V}, 1, \mathrm{D} \& R \text { vs } \mathrm{V}, 3, \mathrm{D} \& R\end{array}$ & $\begin{array}{r}.03 \\
.54 \\
-.25\end{array}$ & $\begin{array}{l}7 \\
7 \\
3\end{array}$ & $\begin{array}{l}\text { n.s. } \\
\text { n.s. } \\
\text { n.s. }\end{array}$ & $\begin{array}{l}-0.2 \\
-2.4 \\
+1.9\end{array}$ & $\begin{array}{l} \pm 15.8 \\
\pm 10.5 \\
\pm 24.2\end{array}$ \\
\hline N.A. & $\mathrm{O}, 0, \mathrm{D}$ vs $\mathrm{O}, \mathbf{1}, \mathrm{D}$ & 4.94 & 7 & .002 & -36.8 & \pm 17.6 \\
\hline R.H. & $\begin{array}{l}\mathrm{H}, 1, D \& \mathrm{R} \text { vs } \mathrm{H}, 3, D \& \mathrm{R} \\
\mathrm{H}, 1, \mathrm{D} \& R \text { vs } \mathrm{H}, 3, \mathrm{D} \& R\end{array}$ & $\begin{array}{r}.09 \\
-.60\end{array}$ & $\begin{array}{l}7 \\
3\end{array}$ & $\begin{array}{l}\text { n.s. } \\
\text { n.s. }\end{array}$ & $\begin{array}{r}-0.2 \\
+2.4\end{array}$ & $\begin{array}{l} \pm 5.3 \\
\pm 18.7\end{array}$ \\
\hline
\end{tabular}

Note-t value, df, and two-tailed $p$ are given for each comparison of each $S$. When both responses were required, the $t$ value, etc., refer to the response in italics. Also given is the percent change in mean $d_{e}^{\prime}$ performance with an increase in $O U$ and the $95 \%$ confidence interval around the percent change.

values were matched for $\alpha, P O$, and the number of psychophysical judgments required; each pair differed solely in the amount of OU. A positive $t$ (see Table 1) indicates that performance was better at the smaller OU level. Table 1 shows that in only one of six cases was performance significantly better for smaller OU. In the four cases when $\mathrm{OU}$ of 1 was compared with OU of 3 , no differences were seen. Table 1 also provides $95 \%$ confidence intervals for the percent change in mean $d_{e}^{\prime}$ performance with variation in OU. There is at most a $22.3 \%$ decrement in $\mathrm{d}_{\mathbf{e}}^{\prime}$ (with $95 \%$ confidence) when OU

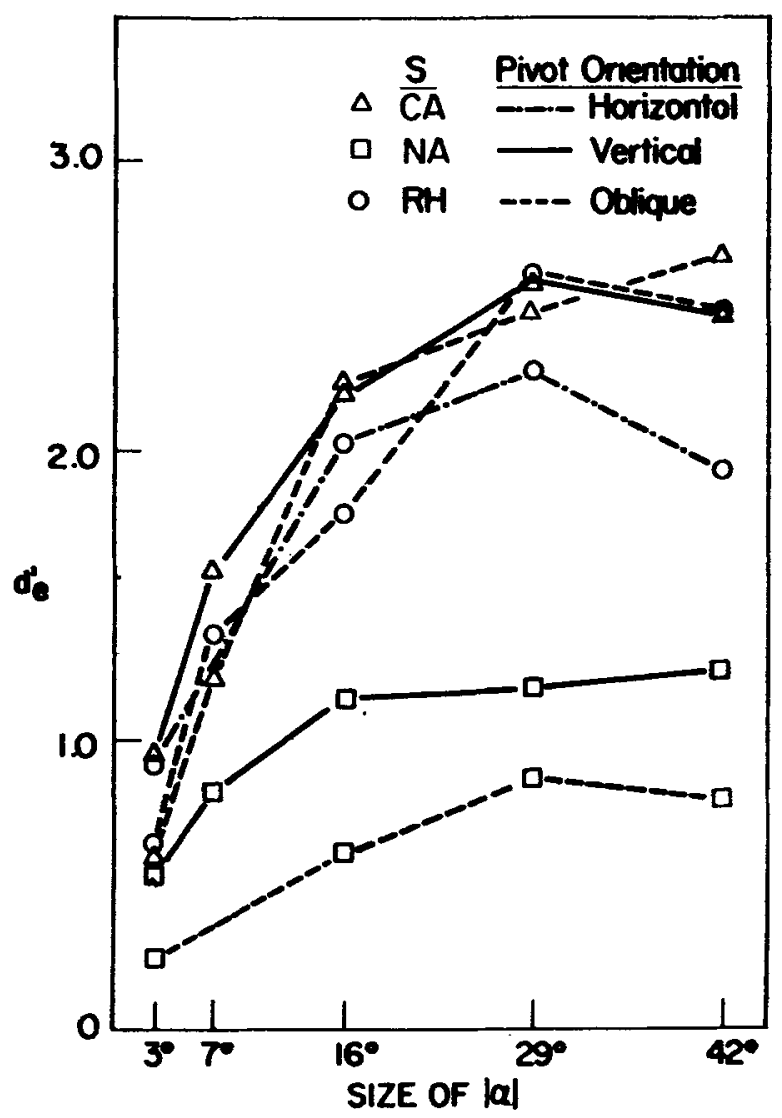

Fig. 1. Recognition performance ( $\left.d_{e}^{\prime}\right)$ as a function of the size of $|\alpha|$ for each $S$ at each PO condition. increased from 1 to 3 . The percent decrease in $d_{e}^{\prime}$ as $O U$ increased from 0 to 1 was quite different for C.A., $\leqslant 16.0 \%$, than for N.A., $\leqslant 54.4 \%-95 \%$ confidence in both cases. For purposes of comparison, in the auditory frequency experiment cited above, Veniar (1958) found a mean drop in d' detection performance of $45.8 \%$ as the number of possible frequencies increased from one to two, and of $35.8 \%$ as the number increased from two to eight.

In considering recognition performance as a function of $\alpha$, the arithmetic mean of $d_{\mathbf{e}}^{\prime}$ was taken over OU of 1 and 3 , weighting each $d_{e}^{\prime}$ according to the number of signal trials on which it was based. The resultant data are plotted in Fig. 1. Performance improves along all six curves as $\alpha$ increases from 3 to $29 \mathrm{deg}$. In general, recognition $d_{e}^{\prime}$ decreases by at least $50 \%$ as $\alpha$ goes from 29 to $3 \mathrm{deg}$. Figure 1 also indicates for the data of N.A. an unexpected difference between recognition about the vertical PO and recognition about the oblique PO.

Turning to the direct comparison between detection and recognition in the condition when both responses were required, Fig. 2 contains the relevant data. Each point consists of plotting, for each $S$ separately, recognition $d_{e}^{\prime}$ and mean detection $d_{e}^{\prime}$ (for $S+$ and $S-$ ) for the same group of trials, with a given PO, OU ( 1 or $3)$, and $\alpha(3,7,16,29$, or $42 \mathrm{deg})$. Since there were no large systematic changes in the relation between detection and recognition for $|\alpha| \geqslant 16 \mathrm{deg}$, OU, or PU, these parameters were not indicated in Fig. 2 . The graph shows that recognition is poorer than detection for all 15 cases in which $|\alpha|=3$ or $7 \mathrm{deg}$. When $|\alpha| \geqslant 16 \mathrm{deg}$, recognition is superior to detection in 24 of 27 cases.

It was thought that perhaps the use of $\mathrm{d}_{e}^{\prime}$ distorted the results reported above. As a check, the full ROC curves were examined, using the area measure, $A_{\text {s }}$ (Pollack \& Hsieh, 1969; Green, 1964). Findings identical to those using $d_{e}^{\prime}$ were obtained for recognition performance as a function of $\alpha$, for detection and recognition as a function of $O U$, and for the comparison between detection and recognition.

\section{DISCUSSION}

The findings of C.A. and R.H. indicate with $95 \%$ 


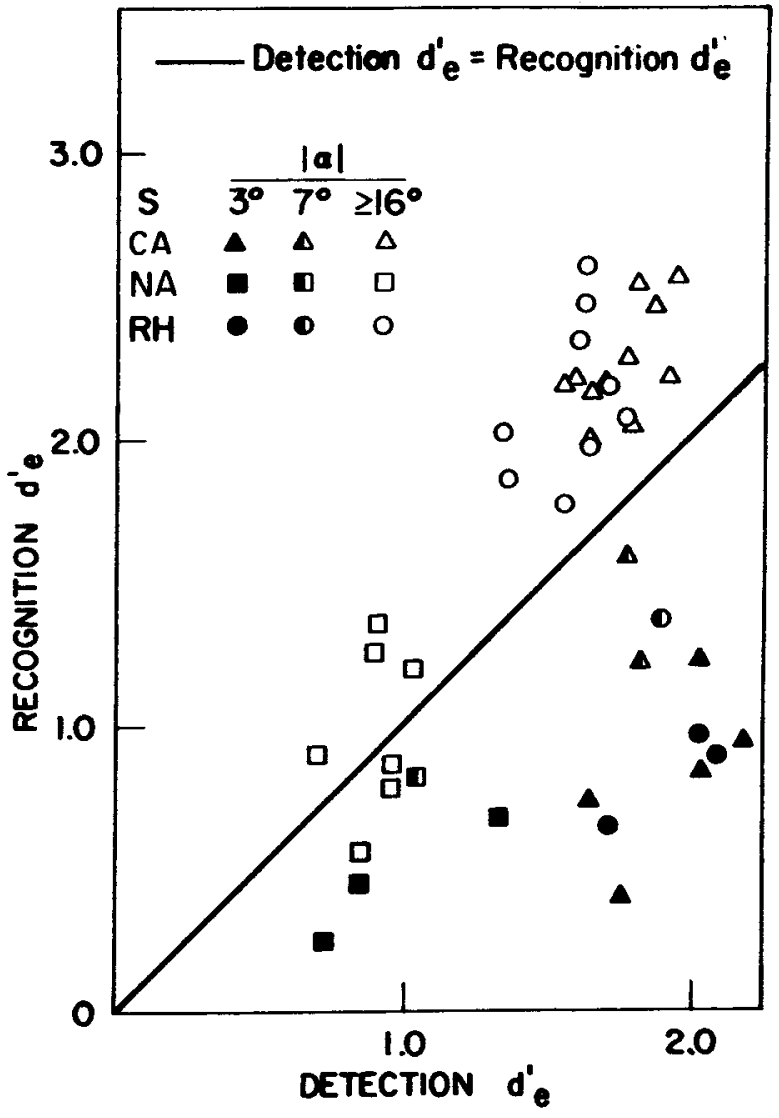

Fig. 2. Relative performance $\left(d_{e}^{\prime}\right)$ on detection and recognition for each $S$ at the specified $|\alpha|$ values. The diagonal line is drawn along the locus of points for which detection and recognition would be equal.

confidence that the effect of increasing OU was at most a $22.3 \%$ decrement in performance. The data of N.A., which point to a larger effect of $\mathrm{OU}$, also showed some discrepancies that call into question their reliability. In general, the present experiment does not yield evidence that varying $\mathrm{OU}$ affects performance to the extent that stimulus uncertainty did in the auditory frequency study of Veniar (1958). One interpretation of the current findings would be that the Ss do not ignore the outputs from irrelevant orientation-sensitive mechanisms, there are no "watching" bands, or, putting it another way, that the outputs of all orientation-sensitive mechanisms are examined by higher order mechanisms.

The measures used were sensitive enough to pick up more than a $50 \%$ drop in recognition with variation in $\alpha$, a result to be expected from the studies cited earlier. The present data suggest that the effect of OU upon performance is miniscule in comparison to the effect of $\alpha$.

The improvement of recognition performance with increasing $\alpha$ is not incompatible with the data of other studies regarding the range of orientation-sensitive mechanisms. The sharpest rise takes place as $\alpha$ increases from 3 to $16 \mathrm{deg}$, with smaller improvement thereafter (see Fig. 1). These findings suggest a sensitivity range of from $30 \mathrm{deg}$ to maybe $40-50 \mathrm{deg}$; the outer limits of the sensitivity range are not clear-cut. But the data in Fig. 1 give a slightly distorted picture of the sensitivity range, since recognition has not been corrected for differences in the detectability of the lines at various orientations. In general, there was only slight change in detection performance with variation in $\alpha .{ }^{4}$ The mean ratio of recognition $d_{e}^{\prime}$ to detection $d_{e}^{\prime}$, taken over the three Ss and the PO conditions for $|\alpha|=3,7,16,29$, and $42 \mathrm{deg}$, is: $.44, .76,1.21,1.26$, and 1.28. This substantiates the notion that the range of the orientation-sensitive mechanisms extends to about $30 \mathrm{deg}$, perhaps a bit more. The suggestion from the studies cited above is that two mechanisms which have preferred orientations separated by much more than $30 \mathrm{deg}$ are independent of one another. The present data do not contradict this assertion (but see Gibson \& Radner, 1937).

Recognition was found to be superior to detection, ${ }^{\mathbf{5}}$ with large $\alpha$, whereas the opposite was the case with small $\alpha$. From the orientation-sensitive mechanism approach it could be said that recognition was better than detection by a factor of about 1.25 when independent orientation-sensitive mechanisms were involved with the alternative stimuli, but that recognition was poorer than detection when some of the same mechanisms were involved.

Perhaps the implicit assumption that the same measure of performance, be it $\mathrm{d}_{\mathrm{e}}^{\prime}$ or $\mathrm{A}_{\mathrm{g}}$, should be used in comparing performance on the two psychophysical tasks is not justified. The recognition of ( $S_{1}$ plus noise) vs ( $S_{2}$ plus noise) is not the same as the detection of ( $S$ plus noise) vs (noise alone). The first involves a difference along some stimulus quality, for example, auditory frequency or visual orientation, whereas the second concerns a difference in something like perceived stimulus intensity. The mechanisms involved in comparing qualitative differences between two stimuli could be quite dissimilar to mechanisms involved in comparing quantitative differences between two stimuli. If so, then the use of the same performance measure for the two tasks is misleading, since it implies that identical mechanisms are involved in both cases. More work is needed toward clarifying the similarity of the mechanisms involved in psychophysical detection and recognition.

\section{REFERENCES}

Antelman, S. M., Olson, R., \& Orbach, J. Orientation-specific mechanisms in the human visual system. Perception \& Psychophy sics, 1969, 6, 206-208.

Atkinson, J. The effect of size, retinal locus, and orientation on the visibility of a single afterimage. Perception \& Psychoph ysics, 1972, 12, 213-217.

Bock, R. D., \& Jones, L. V. The measurement and prediction of judgment and choice. San Francisco: Holden-Day, 1968.

Campbell, F. W., \& Kulikowsiki, J. J. Orientational selectivity of the human visual system. Journal of Physiology, 1966, 187, 437-445.

Coltheart, $M$. Visual feature-analyzers and aftereffects of tilt and curvature. Psychological Review, 1971, 78, 114-121. 
Gibson, J. J., \& Radner, J. Adaptation, aftereffect, and contrast in the perception of tilted lines. Journal of Experimental Psychology, 1937, 20, 453-467.

Gilinsky, A. S. Orientation-specific effects of adapting light on visual acuity. Journal of the Optical Society of America, 1968, 58, 13-17.

Gilinsky, A. S., \& Doherty, R. S. Interocular transfer of orientational effects. Science, 1969, 164, 454-455.

Green, D. M. General prediction relating yes-no and forced choice results. Journal of the Acoustical Society of America, $1964,36,1042(A)$.

Green, D. M., \& Swets, J. A. Signal detection theory and psychophysics. New York: Wiley, 1966.

Houlihan, K., \& Sekuler, R. W. Contour interactions in visual masking. Journal of Experimental Psychology, 1968, 77, 281-285.

Leibowitz, H. W., Myers, N. A., \& Grant, D. A. Radial localization of a single stimulus as a function of luminance and duration of exposure. Journal of the Optical Society of America, 1955, 45, 76-78.

Mayo, T. H., Gilinsky, A. S., \& Jochnowitz, A. Visual masking and tilt aftereffects as a function of tilt and angular separation between test and adaptation line patterns. Proceedings of the 76 th Annual Convention of the American Psychological Association, 1968, 3, 383-386.

Nachmias, J., \& Steinman, R. M. Study of absolute visual detection by the rating-scale method. Journal of the Optical Society of America, 1963,53, 1206-1213.

Ogilvie, J. C., \& Taylor, M. M. Effect of length on the visibility of a fine line. Journal of the Optical Society of America, $1959,49,898-900$.

Parlee, M. B. Visual backward masking of a single line by a single line. Vision Research, 1969, 9, 199-205.

Pollack, I., \& Hsieh. R. Sampling variability of the area under the ROC-curve and of $d_{e}^{\prime}$. Psychological Bulletin, 1969, 71, 161-173.

Rollman, G. B., Nachmias, J. Simultaneous detection and recognition of chromatic flashes. Perception \& Psychophysics, 1972, 12, 309-314.

Sekuler, $R$. W. Spatial and temporal determinants of visual backward masking. Journal of Experimental Psychology, 1965, 70, $401-406$.

Shipley, E. F. Detection and recognition: Experiments and choice models. Journal of Mathematical Psychology, 1965, 2, $277-311$.

Tanner, W. P., Jr. Theory of recognition. Journal of the Acoustical Society of America, 1956, 28, 882-888.

Veniar, F. A. Signal detection as a function of frequency ensemble, II. Journal of the Acoustical Society of America, $1958,30,1075-1078$.

\section{NOTES}

1. Throughout this paper, the terms "horizontal," "vertical," and "oblique" refer to orientations in the fronto-parallel plane- 0,90 , and $45 \mathrm{deg}$, respectively.

2. No systematic differences were found in either detection or recognition as a function of the order of reporting the two judgments when both were required. The data for the two orders of report will not be considered separately.

3 . The curve-fitting procedure used here is formally identical to that deseribed by Bock and Jones (1968). The procedure minimizes the normit chi square that is attributable to discrepancies between the normal deviates reproduced from the model developed by Bock and Jones and those resulting from the empirical response proportions. A check indicated that there were only slight differences in $d_{e}^{\prime}(<.10)$ using the present method as compared to determining $d_{e}^{\prime}$ by fitting the points by eye. I would like to thank J. E. Keith Smith for his help with this part of the data analysis.

4. In general, detection performance did not change as a function of increasing $\alpha$. The meridional effect (see Atkinson, 1972 ) would predict that lines near horizontal or vertical would have higher detection $d_{e}^{\prime}$ than lines near oblique. The data of C.A. were in agreement with this expectation, whereas the data of N.A. and R.H. were not. Ogilvie and Taylor (1959) and Leibowitz, Myers, and Grant (1955) have also reported that some Ss' data do not confirm the effect.

5. The superiority of recognition over detection has been found before in auditory frequency studies by Tanner (1956) and Shipley (1965).

(Received for publication Sep tember 14, 1973; revision received January $15,1974$. ) 